\title{
LMI ROBUSTLY DECENTRALIZED H OUTPUT FEEDBACK CONTROLLER DESIGN FOR STOCHASTIC LARGE-SCALE UNCERTAIN SYSTEMS WITH TIME-DELAYS
}

\author{
Wen-Ben Wu \\ Department of Electrical Engineering, Chin Min Institute of Technology, Miaoli County, Taiwan, R.O.C. Department of \\ Electrical Engineering, Kao Yuan University, Kaohsiung County, Taiwan, R.O.C. \\ Pang-Chia Chen \\ Department of Electrical Engineering, Kao Yuan University, Kaohsiung County, Taiwan, R.O.C. \\ Min-Hsiung Hung \\ Department of Electrical and Electronic Engineering, National Defense University, Taoyuan County, Taiwan, R.O.C. \\ Koan-Yuh Chang \\ Department of Electronic Engineering, Chienkuo Technology University, Changhua, Taiwan, R.O.C. \\ Wen-Jer Chang \\ Department of Marine Engineering, National Taiwan Ocean University, Keelung, Taiwan, R.O.C., \\ wjchang@mail.ntou.edu.tw
}

Follow this and additional works at: https://jmstt.ntou.edu.tw/journal

Part of the Electrical and Computer Engineering Commons

\footnotetext{
Recommended Citation

Wu, Wen-Ben; Chen, Pang-Chia; Hung, Min-Hsiung; Chang, Koan-Yuh; and Chang, Wen-Jer (2009) "LMI ROBUSTLY DECENTRALIZED H OUTPUT FEEDBACK CONTROLLER DESIGN FOR STOCHASTIC LARGE-SCALE UNCERTAIN SYSTEMS WITH TIME-DELAYS," Journal of Marine Science and Technology. Vol. 17: Iss. 1, Article 7. DOI: $10.51400 / 2709-6998.1976$

Available at: https://jmstt.ntou.edu.tw/journal/vol17/iss1/7

This Research Article is brought to you for free and open access by Journal of Marine Science and Technology. It has been accepted for inclusion in Journal of Marine Science and Technology by an authorized editor of Journal of Marine Science and Technology.
} 


\title{
LMI ROBUSTLY DECENTRALIZED $H_{\infty}$ OUTPUT FEEDBACK CONTROLLER DESIGN FOR STOCHASTIC LARGE-SCALE UNCERTAIN SYSTEMS WITH TIME-DELAYS
}

\author{
Wen-Ben $\mathrm{Wu}^{* * * * *}$, Pang-Chia Chen**, Min-Hsiung Hung***, \\ Koan-Yuh Chang****, and Wen-Jer Chang*****
}

Key words: LMI, large-scale uncertain time-delay system, decentralized control, stochastic control.

\begin{abstract}
The present paper investigates the problem of robustly decentralized $H_{\infty}$ output feedback controller design for a class of stochastic large-scale uncertain systems with time-delays. The considered time-delay parameters appear in the interconnections between individual subsystems and uncertainties are allowed to be unstructured but time-varying and norm-bounded. The sufficient conditions of the desired output feedback controller are based on the Lyapunov-Krasovskii stability theory and utilizing the decentralized scheme to be derived in terms of linear matrix inequalities (LMIs). The effectiveness of the proposed approach is illustrated by a numerical example.
\end{abstract}

\section{INTRODUCTION}

Large-scale systems, consisting of a set of interconnected lower-dimension subsystems, are frequently encountered in the real world and include power systems, digital communication networks, flexible manufacturing systems and so on. Owing to the existence of interconnections among subsystems, the controller design of a large-scale system is in general much more difficult than that of individual subsystem. These difficulties motivates the development of decentralized control theory where each subsystem is controlled independently base on its

Paper submitted 09/04/07; accepted 02/25/08. Author for correspondence: Wen-Jer Chang (e-mail: wjchang@mail.ntou.edu.tw).

* Department of Electrical Engineering, Chin Min Institute of Technology, Miaoli County, Taiwan, R.O.C.

** Department of Electrical Engineering, Kao Yuan University, Kaohsiung County, Taiwan, R.O.C.

*** Department of Electrical and Electronic Engineering, National Defense University, Taoyuan County, Taiwan, R.O.C.

**** Department of Electronic Engineering, Chienkuo Technology University, Changhua, Taiwan, R.O.C.

***** Department of Marine Engineering, National Taiwan Ocean University, Keelung, Taiwan, R.O.C. locally available information. Because the advantage of this scheme in controller design is able to reduce complexity and allows the control implementation to be feasible, the problem of decentralized control of large-scale interconnected systems therefore became an attractive topic and many applications have been extensively reported in the literatures [7, 5].

As many of the disturbances acting on the systems are random in nature, the performance analysis must directly address the stochastic aspect of the problem. Stochastic systems have received much attention since stochastic modeling has become to play an important role in many branches of science and engineering applications. Many fundamental results for stochastic large-scale systems have been investigated [13, 2]. On the other hand, delays are generally inherent in many physical systems due to transportation or computation time and uncertainties are unavoidably occurred in many processes, such as plant modeling errors, linearization approximations, exogenous perturbations, and measured noises. Since delay and uncertainty often cause deterioration of system performance and may be a source of instability. Therefore, some significant results including robust stability analysis and decentralized stabilization for uncertain stochastic large-scale time-delay systems have been proposed in [15]. Furthermore, in the recent reports [14, 12], extending to advance the performance of $H_{\infty}$ controllers design via decentralized state feedback have been considered.

Since the state feedback controller design required the complete access to the state vector of the systems. Usually, this is not the case and the state vector cannot be accessed for many reasons well-known in control community. In the case of the state vector not completely accessible, an alternate approach by designing an output feedback controller to stabilize the studied class of systems is considered. Therefore, over the past few years, increasing attention has been given to the problem of constructing $H_{\infty}$ controller with output feedback design. For instance, an overview of linear matrix inequality (LMI) approach to the multi-objective synthesis of linear output feedback controllers for nominal multi-input/multi-output (MIMO) linear time-invariant systems have been investigated in [8]. As is well known, LMI approach due to its computational advantage and simplicity in solving the multi-objective problems such that it 
has become as a powerful formulation and design technique for a variety of linear control problems. Indeed, the controller parameters which satisfy the multi-objective constraint LMIs can be easily found by various efficient convex optimization algorithms. In addition, exploring the Riccati-equation-based approach to design robust observer-based feedback $H_{\infty}$ controller for linear uncertain time-delay systems without interconnections has been proposed in [3]. In [16], an explicit construction of decentralized output feedback $H_{\infty}$ controller is presented using LMI approach for the interconnected time-delay system without uncertainties. Applying generalized inverse theory to design a satisfying multi-objective output feedback control law for the uncertain systems with no interconnections are also addressed in [11]. So far, however, still very few robustly decentralized $H_{\infty}$ output feedback controller (RDHOFC) designs, via LMI approach, are available for the stochastic large-scale uncertain systems with time-delay property.

As the controller design presented in this paper, base on the Lyaunov-Krasovskii functional stability theory [6] and utilizing the decentralized scheme and LMI approach [1, 9, 4], we investigate the problem of developing a RDHOFC for the stochastic large-scale uncertain systems with time-delays. The considered time-delay parameters appear in the interconnections between individual subsystems and uncertainties are allowed to be unstructured but time-varying and norm-bounded. Eventually, the resulting decentralized output feedback controllers can ensure the corresponding overall closed-loop uncertain time-delay systems to achieve the addressed $H_{\infty}$ performance constraint.

This paper is organized as follows. In Section II, gives the problem statement and constraint formulation. In Section III, an algorithm for constructing RDHOFC is developed by using Lyapunov-Krasovskii functional stability theory, decentralized scheme and LMI approach. The effectiveness of the current work is illustrated by a numerical example in Section IV. Finally, some conclusions are given in Section V.

Notation: Throughout this paper, $R^{n}$ denotes the $n$-dimensional Euclidean space; $R^{n \times m}$ is the set of $n \times m$ real matrices. $I$ is the identity matrix with appropriate dimensions; $\operatorname{diag}\{\cdots\}$ stands for the diagonal matrix; The notation $(\cdot)^{T}$ denotes the transpose of the vector or matrix $(\cdot) ;\|\cdot\|$ refers to the Euclidean vector norm; \|\|$_{2}$ represents the usual $L_{2}[0, \infty)$ norm; $L_{2}[0, \infty)$ is the space of square-integrable vector functions over $[0, \infty)$. If both $A$ and $B$ are square matrices with the same dimensions, by $A>B$ (respectively, $A \geq B$ ) we mean $A-B$ is positive definite (respectively, non-negative definite). Moreover, let $\left(\Omega, \mathcal{F}\left(\boldsymbol{Z}_{t}\right)_{t \in \mathbb{R}^{+}}, \mathcal{P}\right)$ be a complete probability space with a filtration $\left(\mathcal{F}_{t}\right)_{t \in \mathbb{R}^{+}}$satisfying the usual conditions (i.e., the filtration contains all $\boldsymbol{P}$-null sets and is right continuous); $\mathbf{E}[\cdot]$ is the expectation operator with respect to some probability measure $P$.

\section{PROBLEM STATEMENT AND FORMULATION}

Consider the stochastic large-scale uncertain time-delay systems which consist of the interconnection of $N$ linear subsystems of the form:

$$
\begin{aligned}
& \dot{x}_{i}(t)=\left(A_{i}+\Delta A_{i}\right) x_{i}(t)+\left(B_{i}+\Delta B_{i}\right) u_{i}(t) \\
&+\sum_{\substack{j=1 \\
j \neq i}}^{N}\left(A_{i j}+\Delta A_{i j}\right) x_{j}\left(t-\tau_{i j}\right)+D_{i} w_{i}(t) \\
& z_{i}(t)= F_{i} x_{i}(t), \\
& y_{i}(t)= C_{i} x_{i}(t), \\
& x_{i}(\theta)= \phi_{i}(\theta), \quad \forall \theta \in\left[-\tau_{i j}, 0\right], \\
& \tau_{i j}>0, \quad i, j=1,2, \cdots, N, \quad j \neq i,
\end{aligned}
$$

where $x_{i}(t) \in R^{n_{i}}, \quad u_{i}(t) \in R^{m_{i}}, \quad z_{i}(t) \in R^{p_{i}}$, and $y_{i}(t) \in R^{q_{i}}$, $i=1,2, \cdots, N$, are the state , control input, controlled output and measurement output of the $i^{\text {th }}$ subsystem, respectively; $w_{i}(t) \in R^{r_{i}}, i=1,2, \cdots, N$, is the white noise input defined on a filtered probability space $\left(\Omega, \mathcal{Z},\left(\mathcal{F}_{t}\right)_{t \in \mathbb{R}^{+}}, \boldsymbol{P}\right)$ and satisfies the following properties:

$$
\begin{aligned}
& \mathbf{E}\left[w_{i}(t)\right]=0, \quad \mathbf{E}\left[x_{i}(0) w_{i}^{T}(t)\right]=0, \\
& \mathbf{E}\left[w_{i}(t) w_{i}^{T}(t)\right]=I, \quad \mathbf{E}\left[w_{i}(t) w_{j}^{T}(t)\right]=0, \\
& i, j=1,2, \cdots, N, \quad j \neq i .
\end{aligned}
$$

Furthermore, in equation ( 1$), \tau_{i j} \geq 0, i, j=1,2, \cdots, N, j \neq i$, is the time-delay existing in the interconnection and $\phi(\theta) \in \tilde{\boldsymbol{C}}\left[-\tau_{i j}, 0\right]$ is the initial condition, where $\tilde{\boldsymbol{C}}\left[-\tau_{i j}, 0\right]$ stands for a space of continuous functions defined on $\left[-\tau_{i j}, 0\right]$. $A_{i}, B_{i}, D_{i}, F_{i}, C_{i}$ and $A_{i j}, i, j=1,2, \cdots, N, j \neq i$, are the known real constant matrices with appropriate dimensions, and $A_{i j}$ are interconnection matrices between the $i^{\text {th }}$ and $j^{\text {th }}$ subsystem. $\Delta A_{i}(t), \Delta B_{i}(t)$ and $\Delta A_{i j}(t), i, j=1,2, \cdots, N, j \neq i$, are matrices representing system time-varying parameter uncertainties which are assumed to be of the form

$$
\begin{aligned}
& \Delta A_{i}(t)=H_{i a} S_{i a}(t) E_{i a}, \\
& \Delta B_{i}(t)=H_{i b} S_{i b}(t) E_{i b}, \\
& \Delta A_{i j}(t)=H_{i j} S_{i j}(t) E_{i j},
\end{aligned}
$$

where $H_{i a}, H_{i b}, H_{i j}, E_{i a}, E_{i b}$, and $E_{i j}$, are known constant matrices; $S_{i a}(t), S_{i b}(t)$, and $S_{i j}(t)$, are unknown real time-varying matrix functions with Lebesgue measurable elements satisfying the following norm-bounded conditions: 


$$
\begin{aligned}
& S_{i a}(t) S_{i a}^{T}(t) \leq I, \\
& S_{i b}(t) S_{i b}^{T}(t) \leq I, \\
& S_{i j}(t) S_{i j}^{T}(t) \leq I, \quad \forall t .
\end{aligned}
$$

Then, $\Delta A_{i}(t), \Delta B_{i}(t)$ and $\Delta A_{i j}(t)$ are said to be admissible if both conditions (3) and (4) hold.

Before formulating the problem to be dealt in this paper, we first introduce the following concepts of robustly stochastic stability in probability for the $i^{\text {th }}$ subsystem (1).

Definition 2.1 [17]: The equilibrium $x_{i}(t)=0$ of the $i^{\text {th }}$ nominal stochastic subsystem (1) is said to be mean-square asymptotically stablizable in probability, via designed decentralized dynamic controller $u_{i}(t)$, if for any scalar $\varepsilon>0$ and initial condition $x_{i}\left(t_{0}\right)>0$, there exists a $\delta=\delta(\varepsilon)$ such that $\mathbf{E}\left[\left\|x_{i}\left(t_{0}\right)\right\|^{2}\right]<\delta$ is satisfied, then both probabilities $\lim _{x\left(t_{0}\right) \rightarrow 0} \mathcal{P}\left\{\sup _{t \geq t_{0}} \mathbf{E}\left\|x_{i}(t)\right\|^{2}>\varepsilon\right\}=0$ and $\mathcal{P}\left\{\lim _{t \rightarrow \infty} \mathbf{E}\left[\left\|x_{i}(t)\right\|^{2}\right]=0\right\}=1$ hold. Furthermore, if the $i^{t h}$ nominal stochastic subsystem (1) is mean-square asymptotically stabilizable in probability, via designed decentralized dynamic controller $u_{i}(t)$, for any time-delays and all admissible uncertainties as defined in both (3) and (4), then the subsystem (1) is said to be robustly stochastically stabilizable in probability.

We now consider a full order dynamic decentralized output feedback control law $u_{i}=G_{i} y_{i}$ which can be represented as the following state-space realization form in (5) for the $i^{\text {th }}$ subsystem [8]:

$$
\begin{aligned}
& \dot{\xi}_{i}(t)=A_{i k} \xi_{i}(t)+B_{i k} y_{i}(t) \\
& u_{i}(t)=C_{i k} \xi_{i}(t)+D_{i k} y_{i}(t), \quad i=1,2, \cdots, N .
\end{aligned}
$$

Applying the designed control law (5) to the system (1), then, the corresponding closed-loop system is given by

$$
\begin{aligned}
\dot{\hat{x}}_{i}(t)= & \left(\hat{A}_{i}+\Delta \hat{A}_{i}\right) \hat{x}_{i}(t)+\hat{D}_{i} w_{i}(t) \\
& +\sum_{\substack{j=1 \\
j \neq i}}^{N}\left(\hat{A}_{i j}+\Delta \hat{A}_{i j}\right) \hat{x}_{i}\left(t-\tau_{i j}\right) \\
z_{i}(t)= & \hat{F}_{i} \hat{x}_{i}(t)
\end{aligned}
$$

where $\hat{x}_{i}(t)=\left[\begin{array}{ll}x_{i}^{T}(t) & \xi_{i}^{T}(t)\end{array}\right]^{T}$, and

$$
\begin{aligned}
& \hat{A}_{i}=\left[\begin{array}{cc}
A_{i}+B_{i} D_{i k} C_{i} & B_{i} C_{i k} \\
B_{i k} C_{i} & A_{i k}
\end{array}\right], \\
& \Delta \hat{A}_{i}=\left[\begin{array}{cc}
\Delta A_{i}+\Delta B_{i} D_{i k} C_{i} & \Delta B_{i} C_{i k} \\
0 & 0
\end{array}\right], \\
& \hat{A}_{i j}=\left[\begin{array}{cc}
A_{i j} & 0 \\
0 & 0
\end{array}\right], \quad \Delta \hat{A}_{i j}=\left[\begin{array}{cc}
\Delta A_{i j} & 0 \\
0 & 0
\end{array}\right], \\
& \hat{D}_{i}=\left[\begin{array}{c}
D_{i} \\
0
\end{array}\right], \quad \hat{F}_{i}=\left[\begin{array}{ll}
F_{i} & 0
\end{array}\right] .
\end{aligned}
$$

In control law (5), $\xi_{i}(t) \in R^{n_{i}}$ denotes the controller state and in equation (7), $A_{i k}, B_{i k}, C_{i k}$ and $D_{i k}, i, j=1,2, \cdots, N$, are unknown controller parameters with appropriate dimensions to be determined.

The purpose of the current paper is based on the decentralized scheme to design a decentralized output feedback control law (5) for the $i^{\text {th }}$ subsystem such that the overall closed-loop system (6) is robustly stochastically stabilizable in probability as defined in Definition 2.1 and satisfies the following $H_{\infty}$ norm performance constraint [16, 17],

$$
\sum_{i=1}^{N} \mathbf{E}\left[\left\|z_{i}(t)\right\|_{2}\right] \leq \sum_{i=1}^{N} \gamma_{i}\left\|w_{i}(t)\right\|_{2}, \quad i=1,2, \cdots, N,
$$

where

$$
\mathbf{E}\left[\left\|z_{i}(t)\right\|_{2}\right]=\mathbf{E}\left[\left(\int_{0}^{\infty} z_{i}^{T}(t) z_{i}(t) d t\right)^{1 / 2}\right],
$$

and the performance level upper bound $\gamma_{i}>0$ can be implemented as a parameter to be minimized during the controller construction.

\section{CONTROLLER DESIGN}

In this section, based on Lyapunov-Krasovskii stability theory, an algorithm for solving the problem of constructing the RDHOFC will be developed by using LMI approach for the stochastic large-scale uncertain systems with time-delays. It can be also considered as designing a robustly stochastic stabilization subject to $H_{\infty}$ norm performance constraint. Before proceeding further, we give the following useful lemma for the proof of this work.

Lemma 3.1 [10]: Let $U, V, W$, and $S(t)$ be real matrices of appropriate dimensions, with $S(t)$ satisfying the normbounded condition $S(t) S^{T}(t) \leq I, \forall t$. Then for any matrix $Q>0$ and scalar $\alpha>0$, such that the following results both (10) and (11) are hold.

$$
\begin{gathered}
Q(U S(t) V)+(U S(t) V)^{T} Q \leq \alpha Q^{T} U U^{T} Q+\alpha^{-1} V^{T} V, \\
U^{T} V+V^{T} U \leq \alpha U^{T} U+\alpha^{-1} V^{T} V .
\end{gathered}
$$

We now define the Lyapunov-Krasvoskii functionnal candidate for the overall interconnected closed-loop system (6) in the following form [15]:

$$
V(\hat{x})=\sum_{i=1}^{N}\left\{\hat{x}_{i}^{T}(t) P_{i c l} \hat{x}_{i}(t)+\sum_{\substack{j=1 \\ j \neq i}}^{N} \int_{t-\tau_{i j}}^{t} \hat{x}_{j}^{T}(\theta) \tilde{P}_{j c l} \hat{x}_{j}(\theta) d \theta\right\},
$$

where $P_{i c l}$ and $\tilde{P}_{j c l}, i, j=1,2, \cdots, N, j \neq i$, are some positive definite matrices such that $V(\hat{x})>0$. Taking expectation of the time derivative of equation (12), we then have 


$$
\begin{aligned}
\mathbf{E} & {\left[\frac{d}{d t} V(\hat{x})\right] } \\
= & \sum_{i=1}^{N} \mathbf{E}\left[\hat{x}_{i}^{T}(t)\left(\hat{A}_{i}^{T} P_{i c l}+P_{i c l} \hat{A}_{i}\right) \hat{x}_{i}(t)\right. \\
& +\hat{x}_{i}^{T}(t)\left(\Delta \hat{A}_{i}^{T} P_{i c l}+P_{i c l} \hat{A}_{i}\right) \hat{x}_{i}(t) \\
& +\hat{x}_{i}^{T} P_{i c l} \hat{D}_{i} w_{i}+w_{i}^{T} \hat{D}_{i}^{T} P_{i c l} \hat{x}_{i} \\
& +\sum_{\substack{j=1 \\
j \neq i}}^{N}\left\{\left(\hat{x}_{i}^{T}(t) P_{i c l} \hat{A}_{i j} \hat{x}_{j}\left(t-\tau_{i j}\right)+\hat{x}_{j}^{T}\left(t-\tau_{i j}\right) \hat{A}_{i j}^{T} P_{i c l} \hat{x}_{i}(t)\right)\right. \\
& \left.+\left(\hat{x}_{i}^{T}(t) P_{i c l} \Delta \hat{A}_{i j} \hat{x}_{j}\left(t-\tau_{i j}\right)+\hat{x}_{j}^{T}\left(t-\tau_{i j}\right) \Delta \hat{A}_{i j}^{T} P_{i c l} \hat{x}_{i}(t)\right)\right\} \\
& \left.+\sum_{\substack{j=1 \\
j \neq i}}^{\mathrm{N}}\left(\hat{x}_{j}^{T}(t) \tilde{P}_{j c l} x_{j}(t)-\hat{x}_{j}^{T}\left(t-\tau_{i j}\right) \tilde{P}_{j c l} \hat{x}_{j}\left(t-\tau_{i j}\right)\right)\right] \\
\triangleq & \sum_{i=1}^{N} \tilde{V}_{i}
\end{aligned}
$$

By Lemma 3.1 and assumption (3), we obtain

$$
\begin{gathered}
\hat{x}_{i}^{T}(t)\left(\Delta \hat{A}_{i}^{T} P_{i c l}+P_{i c l} \Delta \hat{A}_{i}\right) \hat{x}_{i}(t) \\
\leq \hat{x}_{i}^{T}(t)\left(\alpha_{i a} P_{i c l} \tilde{H}_{i a} \tilde{H}_{i a}^{T} P_{i c l}+\alpha_{i a}^{-1} \tilde{E}_{i a}^{T} \tilde{E}_{i a}\right. \\
\left.+\alpha_{i b} P_{i c l} \tilde{H}_{i b} \tilde{H}_{i b}^{T} P_{i c l}+\alpha_{i b}^{-1} \tilde{E}_{i b}^{T} \tilde{E}_{i b}\right) \hat{x}_{i}(t), \\
\hat{x}_{i}^{T}(t) P_{i c l} \Delta \hat{A}_{i j} \hat{x}_{j}\left(t-\tau_{i j}\right)+\hat{x}_{j}^{T}\left(t-\tau_{i j}\right) \Delta \hat{A}_{i j}^{T} P_{i c l} \hat{x}_{i}(t) \\
\leq \alpha_{i j} \hat{x}_{i}^{T}(t) P_{i c l} \tilde{H}_{i j} \tilde{H}_{i j}^{T} P_{i c l} \hat{x}_{i}(t) \\
+\alpha_{i j}^{-1} \hat{x}_{j}^{T}\left(t-\tau_{i j} \tilde{E}_{i j}^{T} \tilde{E}_{i j} \hat{x}_{j}\left(t-\tau_{i j}\right),\right.
\end{gathered}
$$

and for any some symmetric positive matrices $R_{i j}=R_{i j}^{T}>0$, $i, j=1,2, \cdots, N, j \neq i$, it is always truth that

$$
\begin{aligned}
& \hat{x}_{i}^{T}(t) P_{i c l} \hat{A}_{i j} \hat{x}_{j}\left(t-\tau_{i j}\right)+\hat{x}_{j}^{T}\left(t-\tau_{i j}\right) \hat{A}_{i j}^{T} P_{i c l} x_{i}(t) \\
& \leq \hat{x}_{i}^{T}(t) P_{i c l} \hat{A}_{i j} \hat{R}_{i j} \hat{A}_{i j}^{T} P_{i c l} \hat{x}_{i}(t)+\hat{x}_{j}^{T}\left(t-\tau_{i j}\right) \hat{R}_{i j}^{-1} \hat{x}_{j}\left(t-\tau_{i j}\right),
\end{aligned}
$$

where $\alpha_{i a}, \alpha_{i b}$ and $\alpha_{i j}$ are positive real numbers and

$$
\begin{aligned}
& \tilde{H}_{i a}=\left[\begin{array}{c}
H_{i a} \\
0
\end{array}\right], \quad \tilde{H}_{i b}=\left[\begin{array}{c}
H_{i b} \\
0
\end{array}\right], \\
& \tilde{E}_{i a}=\left[\begin{array}{ll}
E_{i a} & 0
\end{array}\right], \quad \tilde{E}_{i b}=\left[\begin{array}{ll}
E_{i b} D_{i k} C_{i} & E_{i b} C_{i k}
\end{array}\right], \\
& \tilde{E}_{i j}=\left[\begin{array}{ll}
E_{i j} & 0
\end{array}\right], \quad \tilde{H}_{i j}=\left[\begin{array}{c}
H_{i j} \\
0
\end{array}\right], \quad \hat{R}_{i j}=\left[\begin{array}{cc}
R_{i j} & 0 \\
0 & 0
\end{array}\right] .
\end{aligned}
$$

Let $\tilde{P}_{j c l}=\alpha_{i j}^{-1} \tilde{E}_{i j}^{T} \tilde{E}_{i j}+\hat{R}_{i j}^{-1}$. Then, follows from (13) to (16), a sufficient condition of robustly asymptotical stabilization can be directly obtained with Lyapunov theory as the following quadratic inequality (18), when $w_{i}(t) \equiv 0$,

$$
\sum_{i=1}^{N} \tilde{V}_{i} \leq \sum_{i=1}^{N} \mathbf{E}\left[\hat{x}_{i}^{T}(t) J_{i c l} \hat{x}_{i}(t)\right]<0,
$$

which implies the following inequality hold,

$$
\begin{aligned}
J_{i c l}= & \hat{A}_{i}^{T} P_{i c l}+P_{i c l} \hat{A}_{i}+\alpha_{i a} P_{i c l} \tilde{H}_{i a} \tilde{H}_{i a}^{T} P_{i c l}+\alpha_{i a}^{-1} \tilde{E}_{i a}^{T} \tilde{E}_{i a} \\
& +\alpha_{i b} P_{i c l} \tilde{H}_{i b} \tilde{H}_{i b}^{T} P_{i c l}+\alpha_{i b}^{-1} \tilde{E}_{i b}^{T} \tilde{E}_{i b} \\
& +\sum_{\substack{j=1 \\
j \neq i}}^{N}\left(P_{i c l} \hat{A}_{i j} \hat{R}_{i j} \hat{A}_{i j}^{T} P_{i c l}+\hat{R}_{j i}^{-1}\right. \\
& \left.+\alpha_{i j} P_{i c l} \tilde{H}_{i j} \tilde{H}_{i j}^{T} P_{i c l}+\alpha_{j i}^{-1} \tilde{E}_{j i}^{T} \tilde{E}_{j i}\right)<0 .
\end{aligned}
$$

Obviously, the resulting sufficient condition of robustly asymptotical stabilization (18) is not capable of rejecting white noise disturbance. On the other hand, based on the result in (19), we will apply the $H_{\infty}$ technique, which is still one of the most popular ways to eliminate the external disturbance in the recently literatures, to solve the problem of designing RDHOFC as presented in the following proposition.

Proposition 3.1: Consider the stochastic large-scale uncertain time-delay systems (1) satisfying the assumption (3). Then the system is robustly stochastically stabilizable in probability with $H_{\infty}$ performance level $\gamma_{i}>0$, via output feedback controller (5), for all admissible uncertainties and any time-delays $\tau_{i j} \geq 0$, if there exist matrices $P_{i c l}=P_{i c l}^{T}>0$ such that the following inequality (20) is satisfied,

$$
\left[\begin{array}{ccc}
J_{i c l} & P_{i c l} \hat{D}_{i} & \hat{F}_{i}^{T} \\
\hat{D}_{i}^{T} P_{i c l} & -\gamma_{i}^{2} I & 0 \\
\hat{F}_{i} & 0 & -I
\end{array}\right]<0,
$$

where $J_{i c l}$ and $\hat{D}_{i}, \hat{F}_{i}$ are previously defined in (19) and (7), respectively.

Proof: The $H_{\infty}$ performance constraint (8) can be rewritten as follows:

$$
\sum_{i=1}^{N} \mathbf{E}\left[\int_{0}^{K}\left(z_{i}^{T}(t) z_{i}(t)-\gamma_{i}^{2} w_{i}^{T}(t) w_{i}(t)\right) d t\right]<0
$$

Define

$$
\begin{aligned}
\Gamma(t)= & \sum_{i=1}^{N}\left\{\mathbf{E}\left[\int_{0}^{\kappa}\left(z_{i}^{T}(t) z_{i}(t)-\gamma_{i}^{2} w_{i}^{T}(t) w_{i}(t)\right) d t\right]+\int_{0}^{\kappa} \tilde{V}_{i} d t\right\} \\
& -\mathbf{E}[V(\hat{x}(\kappa))-V(\hat{x}(0))]
\end{aligned}
$$

subject to the zero initial condition $\hat{x}_{i}(0)=0$, we have

$$
\mathbf{E}[V(\hat{x}(\kappa))-V(\hat{x}(0))]=\sum_{i=1}^{N} \int_{0}^{\kappa} \tilde{V}_{i} d t
$$

such that inequality (24) can be hold by $V(\hat{x})>0$,

$$
\Gamma(t) \leq \sum_{i=1}^{N}\left\{\mathbf{E}\left[\int_{0}^{K}\left(z_{i}^{T}(t) z_{i}(t)-\gamma_{i}^{2} w_{i}^{T}(t) w_{i}(t)\right) d t\right]+\int_{0}^{K} \tilde{V}_{i} d t\right\}
$$

Substituting the expression of $\sum_{i=1}^{N} \tilde{V}_{i}$ as defined in (13) into (24) and combining the condition (21), then the following inequality 
(25) can be obtained by letting $\kappa \rightarrow \infty$,

$$
\sum_{i=1}^{N}\left\{\mathbf{E}\left[\int_{0}^{\infty}\left[\begin{array}{c}
\hat{x}_{i}(t) \\
w_{i}(t)
\end{array}\right]^{T}\left[\begin{array}{cc}
J_{i c l}+\hat{F}_{i}^{T} \hat{F}_{i} & P_{i c l} \hat{D}_{i} \\
\hat{D}_{i}^{T} P_{i c l} & -\gamma_{i}^{2} I
\end{array}\right]\left[\begin{array}{c}
\hat{x}_{i}(t) \\
w_{i}(t)
\end{array}\right] d t\right]\right\}<0 .
$$

It ensures that

$$
\left[\begin{array}{cc}
J_{i c l}+\hat{F}_{i}^{T} \hat{F}_{i} & P_{i c l} \hat{D}_{i} \\
\hat{D}_{i}^{T} P_{i c l} & -\gamma_{i}^{2} I
\end{array}\right]<0 .
$$

Hence, the proof is completed by applying Schur Complement to equation (26).

Note that in the resulting inequality (20), the controller parameters $A_{i k}, B_{i k}, C_{i k}$, and $D_{i k}$ are unknown and occur in nonlinear form, thus the condition (20) cannot be considered as an LMI problem. In the sequel, we shall use a method of changing variables such that (20) is reduced to two LMIs [8]. Therefore, the controller parameters can be solved by LMI approach.

First, partition $P_{i c l}$ and its inverse as

$$
P_{i c l}=\left[\begin{array}{cc}
S_{i} & N_{i} \\
N_{i}^{T} & W_{i}
\end{array}\right], \quad P_{i c l}^{-1}=\left[\begin{array}{cc}
T_{i} & M_{i} \\
M_{i}^{T} & U_{i}
\end{array}\right],
$$

where $S_{i}$ and $T_{i}$ are $R^{n_{i} \times n_{i}}$ and symmetric. Note that the identity $P_{i c l} P_{i c l}^{-1}=I$ gives

$$
M_{i} N_{i}^{T}=I-T_{i} S_{i},
$$

and also infers

$$
P_{i c l}\left[\begin{array}{c}
T_{i} \\
M_{i}^{T}
\end{array}\right]=\left[\begin{array}{l}
I \\
0
\end{array}\right]
$$

which leads to

$$
\varphi_{i}^{T} P_{i c l} \varphi_{i}=\eta_{i}^{T} \varphi_{i}=\left[\begin{array}{cc}
T_{i} & I \\
I & S_{i}
\end{array}\right]
$$

where

$$
\varphi_{i}=\left[\begin{array}{cc}
T_{i} & I \\
M_{i}^{T} & 0
\end{array}\right], \quad \eta_{i}=\left[\begin{array}{cc}
I & S_{i} \\
0 & N_{i}^{T}
\end{array}\right] .
$$

Define the new controller variables as

$$
\begin{gathered}
\hat{A}_{i k}=S_{i}\left(A_{i}+B_{i} D_{i k} C_{i}\right) T_{i}+N_{i} B_{i k} C_{i} T_{i}+S_{i} B_{i} C_{i k} M_{i}^{T}+N_{i} A_{i k} M_{i}^{T} \\
\hat{B}_{i k}=S_{i} B_{i} D_{i k}+N_{i} B_{i k} \\
\hat{C}_{i k}=D_{i k} C_{i} T_{i}+C_{i k} M_{i}^{T} \\
\hat{D}_{i k}=D_{i k}
\end{gathered}
$$

Then, we can summarize the above derivation for RDHOFC design into the following main theorem.
Main theorem: Consider the system (1) satisfying the assumption (3). Then the system is robustly stochastically stabilizable in probability with $H_{\infty}$ performance level $\gamma_{i}>0$, via output feedback controller (5), for all admissible uncertainties and any time-delays $\tau_{i j} \geq 0$, if there exist some matrices $R_{i j}=R_{i j}^{T}>0, T_{i}=T_{i}^{T}>0, S_{i}=S_{i}^{T}>0, \hat{A}_{i k}, B_{i k}, C_{i k}, D_{i k}$, and some positive real numbers $\alpha_{i a}, \alpha_{i b}, \alpha_{i j}, i, j=1,2, \cdots, N$, $j \neq i$, such that the following LMI conditions (36) and (37) are satisfied,

$$
\begin{gathered}
{\left[\begin{array}{cc}
T_{i} & I \\
I & S_{i}
\end{array}\right]>0 \cdot} \\
{\left[\begin{array}{llll}
\Theta_{11} & \Theta_{12} & \Theta_{13} & \Theta_{14} \\
\Theta_{12}^{T} & \Theta_{22} & \Theta_{23} & \Theta_{24} \\
\Theta_{13}^{T} & \Theta_{23}^{T} & \Theta_{33} & \Theta_{34} \\
\Theta_{14}^{T} & \Theta_{24}^{T} & \Theta_{34}^{T} & \Theta_{33}
\end{array}\right]<0,}
\end{gathered}
$$

where

$$
\begin{aligned}
& \Theta_{11}=\left[\begin{array}{cc}
A_{i} T_{i}+B_{i} \hat{C}_{i k}+T_{i} A_{i}^{T}+\hat{C}_{i k}^{T} B_{i}^{T} & \hat{A}_{i k}^{T}+\left(A_{i}+B_{i} D_{i k} C_{i}\right) \\
\hat{A}_{i k}+\left(A_{i}+B_{i} D_{i k} C_{i}\right)^{T} & S_{i} A_{i}+\hat{B}_{i k} C_{i}+A_{i}^{T} S_{i}+C_{i}^{T} \hat{B}_{i k}^{T}
\end{array}\right], \\
& \Theta_{12}=\left[\begin{array}{cc}
\widehat{T}_{i} & \widehat{A}_{i} \\
I & S_{i} \hat{A}_{i}
\end{array}\right], \quad \Theta_{13}=\left[\begin{array}{cccc}
H_{i a} & H_{i b} & T_{i} E_{i a}^{T} & \hat{C}_{i k}^{T} E_{i b}^{T} \\
S_{i} H_{i a} & S_{i} H_{i b} & E_{i a}^{T} & \left(E_{i b} D_{i k} C_{i}\right)^{T}
\end{array}\right], \\
& \Theta_{14}=\left[\begin{array}{cccc}
\hat{H}_{i} & T_{i} \hat{E}_{i}^{T} & D_{i} & \left(F_{i} T_{i}\right)^{T} \\
S_{i} \hat{H}_{i} & \hat{E}_{i}^{T} & S_{i} D_{i} & F_{i}^{T}
\end{array}\right],
\end{aligned}
$$

$\widehat{T}_{i}=\left[\begin{array}{lll}T_{i} & \cdots & T_{i}\end{array}\right], \quad \widehat{E}_{i}=\left[\begin{array}{lll}E_{1 i}^{T} & \cdots & E_{1 N}^{T}\end{array}\right]$,

$\widehat{A}_{i}=\left[\begin{array}{lll}A_{i 1} & \cdots & A_{i N}\end{array}\right], \quad \hat{H}_{i}=\left[\begin{array}{lll}H_{i 1} & \cdots & H_{i N}\end{array}\right]$,

$\Pi_{i r}=\operatorname{diag}\left\{R_{i 1} \quad \cdots \quad R_{i N}\right\}$,

$\Pi_{\ell i}=\operatorname{diag}\left\{R_{1 i} \quad \cdots \quad R_{N i}\right\}$,

$\Upsilon_{i r}=\operatorname{diag}\left\{\alpha_{i 1} I \quad \cdots \quad \alpha_{i N} I\right\}$,

$\Upsilon_{\ell i}=\operatorname{diag}\left\{\alpha_{1 i} I \quad \cdots \quad \alpha_{N i} I\right\}$,

$i, r, \ell=1,2, \cdots, N, \quad r, \ell \neq i$. 
Moreover, the controller parameters $A_{i k}, B_{i k}, C_{i k}$ and $D_{i k}$ can be computed using (32) to (35).

Proof: Pre- and post-multiplying $\operatorname{diag}\left\{\begin{array}{lll}\varphi_{i}^{T} & I & I\end{array}\right\}$, $\operatorname{diag}\left\{\varphi_{i} \quad I \quad I\right\}$, respectively, for the both sides of (20) and considering the change of controller variables (32) to (35), then (37) can be obtained by applying Schur Complement to (20). Hence, the proof is complete.

\section{NUMERICAL EXAMPLE}

A numerical example to demonstrate the effectiveness of the proposed RDHOFC design for the stochastic large-scale uncertain time-delay systems is given in this section. Consider the stochastic uncertain time-delay system consisting of two subsystems as follows:

The $1^{\text {st }}$ subsystem:

$$
\begin{aligned}
\dot{x}_{1}(t)= & \left(A_{1}+\Delta A_{1}\right) x_{1}(t)+\left(B_{1}+\Delta B_{1}\right) u_{1}(t) \\
& +\left(A_{12}+\Delta A_{12}\right) x_{2}\left(t-\tau_{12}\right)+D_{1} w_{1}(t), \\
z_{1}(t)= & F_{1} x_{1}(t), \\
y_{1}(t)= & C_{1} x_{1}(t),
\end{aligned}
$$

The $2^{\text {nd }}$ subsystem:

$$
\begin{aligned}
\dot{x}_{2}(t)= & \left(A_{2}+\Delta A_{2}\right) x_{2}(t)+\left(B_{2}+\Delta B_{2}\right) u_{2}(t) \\
& +\left(A_{21}+\Delta A_{21}\right) x_{1}\left(t-\tau_{21}\right)+D_{2} w_{2}(t), \\
z_{2}(t)= & F_{2} x_{2}(t), \\
y_{2}(t)= & C_{2} x_{2}(t),
\end{aligned}
$$

where the states $x_{1}(t)=\left[\begin{array}{ll}x_{11}(t) & x_{12}(t)\end{array}\right]^{T}$, $x_{2}(t)=\left[\begin{array}{ll}x_{21}(t) & x_{22}(t)\end{array}\right]^{T}$ and the system matrices $A_{1}=\left[\begin{array}{cc}0 & 1 \\ -2 & 3\end{array}\right]$, $\Delta A_{1}=\left[\begin{array}{cc}r_{1}(t) & 0 \\ 0 & 0\end{array}\right], \quad B_{1}=\left[\begin{array}{l}0 \\ 1\end{array}\right], \quad \Delta B_{1}=\left[\begin{array}{c}0 \\ v_{1}(t)\end{array}\right], \quad D_{1}=\left[\begin{array}{c}0.1 \\ 0\end{array}\right]$, $A_{12}=\left[\begin{array}{cc}0 & 0 \\ 0.2 & 1\end{array}\right], \quad \Delta A_{12}=\left[\begin{array}{cc}0 & \mu_{12}(t) \\ 0 & 0\end{array}\right], \quad F_{1}^{T}=\left[\begin{array}{l}1 \\ 0\end{array}\right], \quad C_{1}^{T}=\left[\begin{array}{l}1 \\ 1\end{array}\right]$, $A_{2}=\left[\begin{array}{cc}0 & 1 \\ -4 & 5\end{array}\right], \Delta A_{2}=\left[\begin{array}{cc}r_{2}(t) & 0 \\ 0 & 0\end{array}\right], \quad B_{2}=\left[\begin{array}{l}1 \\ 0\end{array}\right], \Delta B_{2}=\left[\begin{array}{c}v_{2}(t) \\ 0\end{array}\right]$, $D_{2}=\left[\begin{array}{c}0.25 \\ 0.5\end{array}\right], A_{21}=\left[\begin{array}{cc}1 & 0.3 \\ 0 & 0\end{array}\right], \Delta A_{21}=\left[\begin{array}{cc}0 & 0 \\ \mu_{21}(t) & 0\end{array}\right], F_{2}^{T}=\left[\begin{array}{l}1 \\ 0\end{array}\right]$, $C_{2}^{T}=\left[\begin{array}{l}1 \\ 1\end{array}\right]$, in which $\left|r_{i}(t)\right| \leq 1,\left|v_{i}(t)\right| \leq 1$ and $\left|\mu_{i j}(t)\right| \leq 1$, respectively.

Suppose that $x_{1}(0)=\left[\begin{array}{ll}4, & 7\end{array}\right]^{T}, x_{2}(0)=\left[\begin{array}{ll}5, & 8\end{array}\right]^{T}$, the white noises $w_{1}(t), w_{2}(t)$ satisfying the properties (2), and the time-delay parameters given as $\tau_{12}=\tau_{21}=5$. Then, the desired output feedback controller parameters $A_{i k}, B_{i k}, C_{i k}$, and $D_{i k}$ can be carried out as follows:
Step 1: From assumption (3), the various known matrices are: $H_{1 a}=H_{12}=H_{2 a}=H_{2 b}=\left[\begin{array}{ll}1 & 0 \\ 0 & 0\end{array}\right] \quad, \quad H_{21}=H_{1 b}=\left[\begin{array}{ll}0 & 0 \\ 0 & 1\end{array}\right]$, $E_{21}=\left[\begin{array}{ll}0 & 0 \\ 1 & 0\end{array}\right], \quad E_{1 b}=\left[\begin{array}{l}0 \\ 1\end{array}\right], \quad E_{2 b}=\left[\begin{array}{l}1 \\ 0\end{array}\right], \quad E_{12}=\left[\begin{array}{ll}0 & 1 \\ 0 & 0\end{array}\right]$, $E_{1 a}=E_{2 a}=\left[\begin{array}{ll}1 & 0 \\ 0 & 1\end{array}\right], S_{i a}(t)=\operatorname{diag}\left\{r_{i}(t)\right\}, S_{i b}(t)=\operatorname{diag}\left\{v_{i}(t)\right\}$, $S_{i j}(t)=\operatorname{diag}\left\{\mu_{i j}(t)\right\}, i, j=1,2, \quad j \neq i$.

Step 2: The LMI optimization matrix variables $T_{i}$ and $S_{i}$, $i=1,2$, that achieve the addressed $H_{\infty}$ performance constraint for the closed-loop systems of (42) and (43) can be solved by using GEVP method in the MATLAB LMI control toolbox subject to the LMI conditions (36) and (37) as

$$
\begin{aligned}
& T_{1}=\left[\begin{array}{cc}
0.4154 & -0.1310 \\
-0.1310 & 0.9156
\end{array}\right], \\
& S_{1}=\left[\begin{array}{cc}
4.9937 & 0.6265 \\
0.6265 & 13.9144
\end{array}\right] ; \\
& T_{2}=\left[\begin{array}{ll}
2.3195 & 1.3474 \\
1.3474 & 0.9485
\end{array}\right], \\
& S_{2}=10^{3} \times\left[\begin{array}{ll}
0.0048 & 0.0205 \\
0.0205 & 3.1760
\end{array}\right],
\end{aligned}
$$

with optimal performance values

$$
\left(\gamma_{1}\right)_{\min }=0.2231, \quad\left(\gamma_{2}\right)_{\min }=0.5927 .
$$

Step 3: The matrices $M_{i}$ and $N_{i}, i=1,2$, can be solved by applying the singular value decomposition method to compute matrices $I-T_{i} S_{i}$. Thus, using equations (32) to (35), the desired output feedback controller parameters $A_{i k}, B_{i k}, C_{i k}$ and $D_{i k}, i=1,2$, for the closed-loop systems of (42) and (43) can be respectively obtained as

$$
\begin{aligned}
& A_{1 k}=\left[\begin{array}{ll}
780.4068 & -82.9767 \\
125.5511 & -13.1220
\end{array}\right], \quad B_{1 k}=\left[\begin{array}{c}
-607.5679 \\
-87.7334
\end{array}\right], \\
& C_{1 k}=\left[\begin{array}{ll}
-10.0646 & -0.1539
\end{array}\right], \quad D_{1 k}=0.1659 \text {; } \\
& A_{2 k}=10^{4} \times\left[\begin{array}{cc}
0.1882 & -0.0109 \\
-2.2976 & -2.8069
\end{array}\right], \quad B_{2 k}=10^{4} \times\left[\begin{array}{c}
-0.3270 \\
2.5759
\end{array}\right], \\
& C_{2 k}=\left[\begin{array}{ll}
-0.2068 & 9.5669
\end{array}\right], \quad D_{2 k}=-0.3023 .
\end{aligned}
$$

Step 4: The complete dynamic output feedback control laws for each subsystem are then

$$
\begin{aligned}
& \dot{\xi}_{1}(t)=\left[\begin{array}{ll}
780.4068 & -82.9767 \\
125.5511 & -13.1220
\end{array}\right] \xi_{1}(t)+\left[\begin{array}{c}
-607.5679 \\
-87.7334
\end{array}\right] y_{1}(t), \\
& u_{1}(t)=\left[\begin{array}{ll}
-10.0646 & -0.1539
\end{array}\right] \xi_{1}(t)+0.1659 y_{1}(t),
\end{aligned}
$$




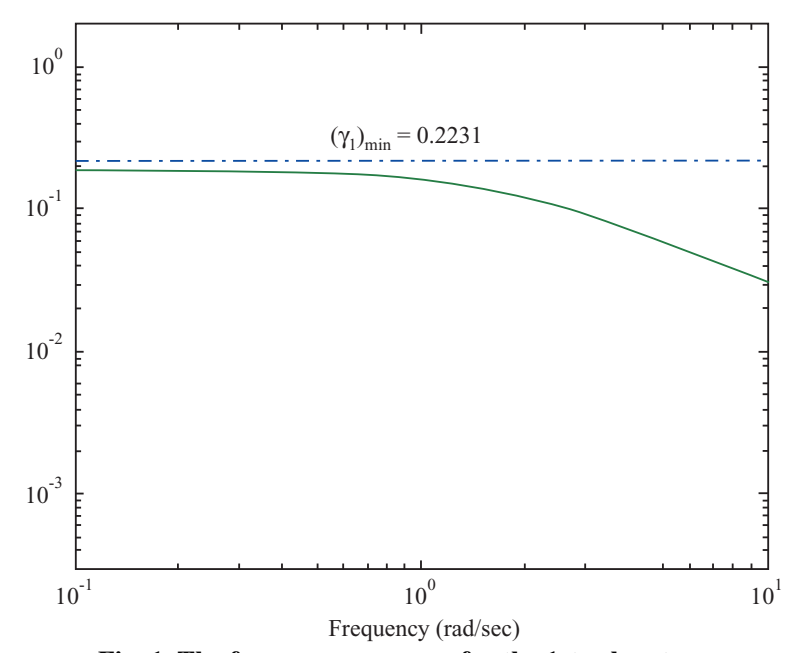

Fig. 1. The frequency response for the 1st subsystem.

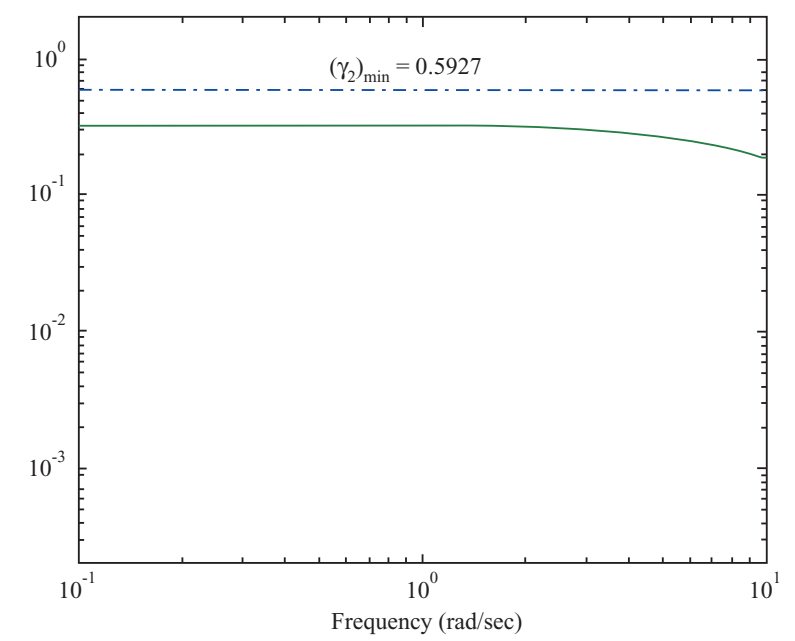

Fig. 2. The frequency response for the 2nd subsystem.
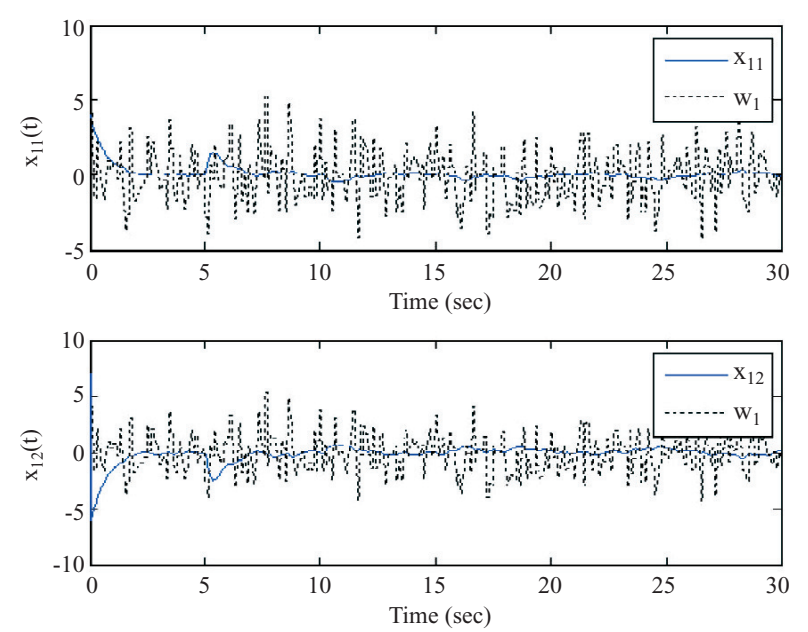

Fig. 3. The time response for state $x_{1}(t)$ of the 1 st subsystem.
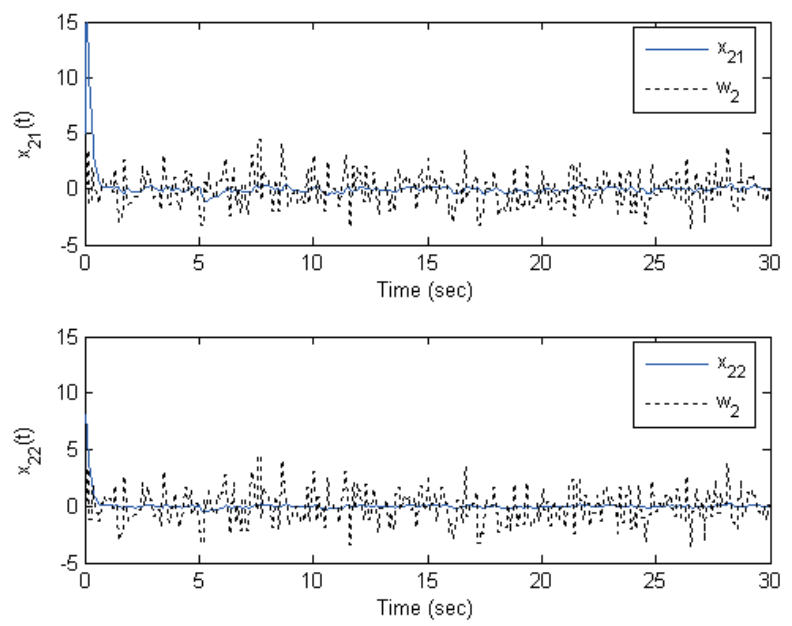

Fig. 4. The time response for state $x_{2}(t)$ of the 2 nd subsystem.

$$
\begin{aligned}
\dot{\xi}_{2}(t)= & 10^{4} \times\left[\begin{array}{cc}
0.1882 & -0.0109 \\
-2.2976 & -2.8069
\end{array}\right] \xi_{2}(t) \\
& +10^{4} \times\left[\begin{array}{c}
-0.3270 \\
2.5759
\end{array}\right] y_{2}(t) \\
u_{2}(t)= & {[-10.0646-0.1539] \xi_{2}(t)-0.3023 y_{2}(t) . }
\end{aligned}
$$

Substituting the control laws (48) and (49) into the corresponding subsystems (42) and (43). Then, the frequency responses of each subsystem are shown in Fig. 1 to Fig. 2, respectively. In which the dotted lines denote the designed upper bounds and solid lines denote the actual value of $H_{\infty}$ norm subject to frequency changed. From Figs. 1 and 2, one knows that the $H_{\infty}$ norm performance specifications (45) are well satisfied. Furthermore, the time responses of each subsystem are shown in Figs. 3 and 4 where the dotted lines are the zero mean, unitary variance noise input sequences $w_{1}(t)$ and $w_{2}(t)$ generated by the MATLAB randn command, and solid lines stand for the states $x_{1}(t)$ and $x_{2}(t)$ response.

\section{CONCLUSION}

The present paper has studied the problem of RDHOFC design for the stochastic large-scale uncertain systems with time-delays. It has been shown that the RDHOFC is developed via a set of linear matrix inequalities is solvable. Ultimately, a numerical example has shown the effectiveness of the proposed approach. In the further study, the result of the current paper therefore can be considered as a useful foundation for solving some stochastic multi-objective control problems.

\section{REFERENCES}

1. Boyd, S., Ghaoui, L. E., Feron, E., and Balakrishnan, V., Linear Matrix Inequality in System and Control Theory, SIAM, Philadelphia, pp. 7-35 (1994).

2. Chang, K. Y. and Chen, P. C., "Multi-objective state feedback control for 
stochastic large-scale systems," Journal of Chinese Institute of Electrical Engineering, Vol. 10, No. 3, pp. 305-314 (2003).

3. Choi, H. H. and Chung, M. J., "Robust observer-based $H_{\infty}$ controller design for linear uncertain time-delay systems," Automatica, Vol. 33, No. 91, pp. 1749-1752 (1997).

4. Gahinet, P., Nemirovski, A., Laub, A. J., and Chilali, M., LMI Control Toolbox for Use with MATLAB, The Math Work Inc., Natick, Massachusetts, p. 4-1 to p. 5-6 (1995).

5. Jamshidi, M., Large-scale System: Modeling, Control, and Fuzzy Logic, Prentice-Hall, Inc., New Jersey, pp. 229-280 (1997).

6. Kharitonov, V. L. and Zhabko, A. P., "Lyapunov-Krasovskii approach to the robust stability analysis of time-delay systems," Automatica, Vol. 39, No. 1, pp. 15-20 (2003).

7. Sandell, N. R., Varaiya, P., Athans, M., and Safonov, M. G., "Survey of decentralized control method for large-scale system," IEEE Transactions on Automatic Control, Vol. 23, No. 2, pp. 108-128 (1978).

8. Scherer, C., Gahinet, P., and Chilali, M., "Multiobjective output-feedback control via LMI optimization," IEEE Transactions on Automatic Control, Vol. 42, No. 7, pp. 896-911 (1997).

9. Vandenberghe, L. V. and Balakrishnan, V. K., "Algorithm and software for LMI problems in control," IEEE Control Systems Lecture Notes, Vol. 17, No. 1, pp. 89-95 (1997).

10. Wang, Y., Xie L. and De Souza, C. E., "Robust control of a class of uncertain nonlinear system," System and Control Letters, Vol. 19, No. 1, pp. 139-149 (1992).
11. Wang, Z. and Ho, W. C., "Output feedback robust $H_{\infty}$ control with D-stability and variance constraints: parametrization approach," Journal of Dynamical and Control Systems, Vol. 11, No. 2, pp. 263-280 (2005).

12. Wu, W. B., Chang, K. Y., Chen, G., Chang, Y. H., and Chen, P. C., "Design of multi-objective robust decentralized controller for stochastic large-scale system using LMI," Proceeding of 2005 CACS Automatic Control Conference, Tainan, Taiwan, R. O. C., p. d9, J00001d (2005).

13. Wu, W. B., Chen, P. C., Chen, G., Chang, K. Y., and Chang, Y. H., "Multiobjective state-feedback control for stochastic large-scale system via LMI approach," Journal of Chung Cheng Institute of Technology, Vol. 34, No. 2, pp. 1-19 (2006).

14. Wu, W. B., Chen, P. C., Chen, G., and Chang, Y. H., "LMI decentralized controller design for stochastic large-scale time-delay systems," Proceeding of the 2004 IEEE International Conference on Control Applications, Taipei, R. O. C., pp. 632-637 (2004).

15. Xie, S. and Xie, L., "Stabilization of class of uncertain large-scale stochastic system with time delay," Automatica, Vol. 36, No. 1, pp. 161-167 (2000).

16. Xie, S., Xie, L., and Wen, C., "Decentralized $H_{\infty}$ output feedback control of interconnected time-delay systems," Proceedings of the American Control Conference, Chicago, Illinois, pp. 824-828 (2000).

17. Xu, S. and Chen, T., "Robust $H_{\infty}$ control for uncertain stochastic systems with state delay," IEEE Transactions on Automatic Control, Vol. 47, No. 12 , pp. 2089-2002 (2002). 